\title{
Grape Seeds the Nontraditional Source for Achieving Bio-Nanomaterials Addressing Antibacterial, Anticancer and Antidiabetic Functions
}

ISSN: 2576-8840

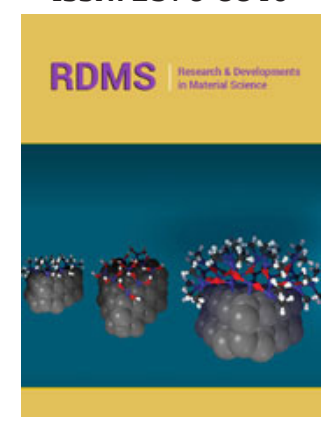

*Corresponding author: Wafa I AbdelFattah, Refractories, Ceramics and Building Materials DeptBiomaterials Group, National Research Centre, Egypt

Submission: 眥 November 23, 2020

Published: 阱December 21, 2020

Volume 14 - Issue 5

How to cite this article: Wafa I AbdelFattah, Waleed Mosaad. Grape Seeds the Nontraditional Source for Achieving BioNanomaterials Addressing Antibacterial, Anticancer and Antidiabetic Functions. Res DevMaterialSci. 14(5).RDMS.000846.2020. DOI: 10.31031/RDMS.2020.14.000846

Copyright@ Wafa I Abdel-Fattah. This article is distributed under the terms of the Creative Commons Attribution 4.0 International License, which permits unrestricted use and redistribution provided that the original author and source are credited.

\author{
Wafa I Abdel-Fattah ${ }^{1 *}$ and Waleed Mosaad $^{2}$ \\ ${ }^{1}$ Refractories, Ceramics and Building Materials DeptBiomaterials Group, National Research \\ Centre, Egypt \\ ${ }^{2}$ Spectroscopy Dept National Research Centre, Egypt
}

\section{Mini Review}

Grape seeds extract contains high concentrations of vitamin E, linoleic acid and flavonoids. Recently, standard grape seed extracts are reported for the treatment of a wide range of health problems concerned with free radical damage, including diabetes, heart disease, and cancer. Also, grape seeds extract can be used as a dietary supplement for various conditions, including venous insufficiency (when veins have problems sending blood from legs back to heart), reducing inflammation and promoting wound healing [1]. Furthermore, Grape seed extract has also been shown to protect against bacterial infections, such as Staphylococcus aureus. Some studies, mostly in animals, support these uses [2].

The new prevalent nutraceuticals assist the process of some diseases prevention. Grapes contain numerous phenolics which assist in protecting against resulting lipid peroxidation. Chronic diseases developments implicated by lipid peroxidation resulting from damaged DNA free radicals are protected by fruit phenolics with its inherited antioxidant capacity. Phenolic extraction from fruits, vegetables, nuts grains, and agricultural by-products is becoming more prevalent for the manufacture of functional foods and nutraceuticals to assist in the prevention of such diseases (food additives and dietary supplements) [3].

Grape Seed Extract (GSE) (Vitis vinifera) is considered the most powerful antioxidants, due to its high levels of flavonoids, vitamin C, and vitamin E. GSE protects cells by regulating cell oxidative damage, reducing organ injury, improving the balance between oxidants and antioxidants, and reducing the release of inflammatory mediators as well [4]. Additionally, GSE has been reported to exert anti-carcinogenic effects [5]. About $60 \%$ to $70 \%$ of grape polyphenols are found in the seeds. These polyphenols are commonly known as proanthocyanidins. Proanthocyanidins obtained from grape seeds have been proven to protect against UV light-induced carcinogenesis, stop immune suppression, enhance interleukin (IL)12, and decrease IL-10.

Anthocyanins were isolated from grape pomace with methanol utilizing ultra-sonication. It was possible to isolate 14 anthocyanin monomers in a single step. An effective method for semi-preparative isolation of grape pomace anthocyanins was developed. Anthocyanins were extracted with methanol along with ultra-sonication. The crude extract was purified using Amberlite XAD-7HP column chromatography. Semi-preparative HPLC yielded 14 anthocyanin monomers with high purity $(\geq 90 \%)$ in a single-step. This procedure has implications for the industrial production of anthocyanins (Figure 1); [6]. 


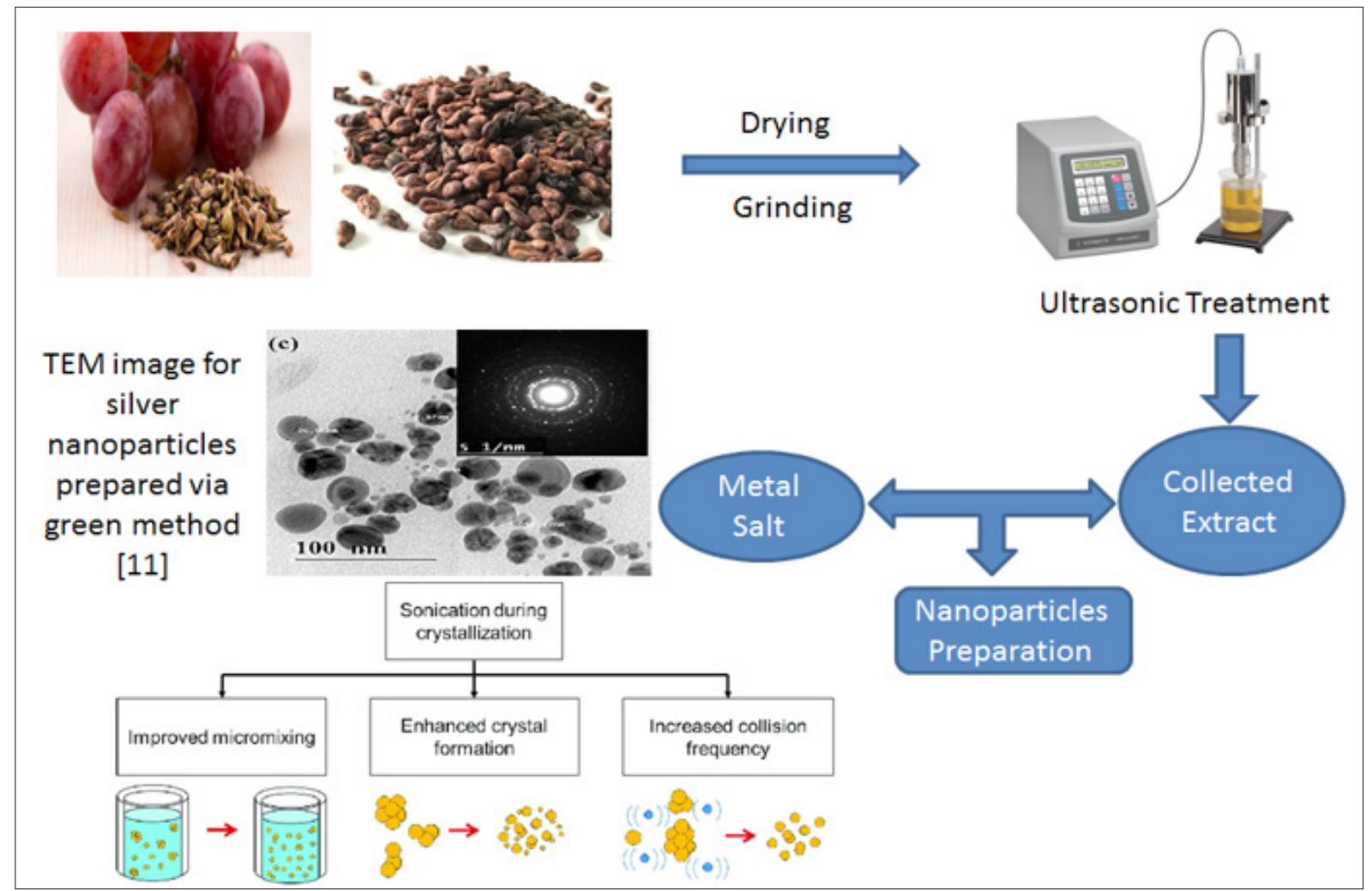

Figure 1: Schematic overview of the proposed mechanism by which sonication inhibits agglomeration during crystallization [6].

Grape (Vitis vinifera L.) pomace, a byproduct of red wine production, is a good source for the production of anthocyanins. An effective simple method for semi-preparative isolation of anthocyanins from grape pomace was developed. Ultra-sonication with acidified $\mathrm{MeOH}$ was used to extract anthocyanin. $50 \mathrm{~g}$ grape pomace produced $56.15 \mathrm{mg}$ of total anthocyanins. Crude extracts were purified by XAD-7HP column chromatography, followed by isolation of the anthocyanin mixtures using semi-preparative HPLC, and subsequent identification of the anthocyanin monomers by HPLC-DAD-MS/MS. Fourteen anthocyanins were isolated with high purities $(\geq 90 \%)$, including non-acylated and acylated anthocyanin's, along with their cis and trans isomers. The primary anthocyanin monomers in grapes were isolated simultaneously using a single-step semi-HPLC procedure. The achieved findings contribute to more research on anthocyanin monomers and grape pomace profitable utilization [7].

A 2019 review of 15 studies involving 825 participants suggested that grape seed extract might help lower levels of LDL cholesterol, total cholesterol, triglycerides, and the inflammatory marker C-reactive protein [2]. Nanotechnology research, recently, deals with the development of environmental-friendly processes for the synthesis of stable nanoparticles, with well-defined shapes, and controlled narrow sizes. A very promising prospect of nanoparticles is its application for targeting drug delivery while "multi-targeting" is essential in several disease analyses. Green biosynthesis of silver nanoparticles was reported using Curcuma longa tuber powder [8].

Recently, the synthesis of metal nanoparticles using plant extracts as the reducing and stabilizing agents is one of the most widely used green techniques. A variety of plants have been successfully utilized for synthesizing metal nanoparticles. Grape Seeds Extraction (GSE) was used to produce plenty of metal nanoparticles such as silver and gold nanoparticles $[9,10]$.

Many factors are controlling the preparation process of nanoparticles using grape seeds extract such as metal salt concentration, the ratio between GSE and metal salts, the reaction temperature, and the reaction $\mathrm{pH}$ value. All those factors influence the preparation process which affects size, shape and antimicrobial functions of the achieved nanoparticles $[11,12]$.

\section{Acknowledgement}

The authors acknowledge the financial support of the Academy of Scientific Research and Technology for the bilateral Egyptian/ Indian project entitled 'Metal cross-linked oxygenated multi-layer polymeric scaffold for diabetic foot ulcer wound healing. Special thanks are due to the National Research Centre for hosting the project. 


\section{References}

1. Zhang H, Liu S, Li L, Liu S, Mi J, Tian G (2016) The impact of grape seed extract treatment on blood pressure changes: A meta-analysis of 16 randomized controlled trials. Med 95(33): 4247.

2. Asbaghi O, Nazarian B, Reiner Z, Amirani E, Kolahdooz F, et al. (2020) The effects of grape seed extract on glycemic control, serum lipoproteins, inflammation, and body weight: A systematic review and meta-analysis of randomized controlled trials. Phyther Res 34(2): 239-253.

3. Kwiatkowski M, Kravchuk O, Skouroumounis GK, Taylor DK (2020) Response surface parallel optimization of extraction of total phenolics from separate white and red grape skin mixtures with microwaveassisted and conventional thermal methods. J Clean Prod 251.

4. Qlayel A, Alkhedaide H, Aq A (2015) The anti-inflammatory effect of grape seed extract in rats exposed to Cadmium Chloride toxicity 3: 298305.

5. Çetin A, Kaynar L, Koçyiğit I, Hacioğlu SK, Saraymen R, et al. (2008) The effect of grape seed extract on radiation-induced oxidative stress in the rat liver. Turkish J Gastroenterol 19(2): 92-98.

6. Ali GW, El-Hotaby W, Hemdan B, Abdel-Fattah WI (2018) Thermosensitive chitosan/phosphate hydrogel-composites fortified with Ag versus Ag@ Pd for biomedical applications. Life Sci 194: 185-195.
7. Zhao X, Zhang SS, Zhang XK, He F, Duan CQ (2020) An effective method for the semi-preparative isolation of high-purity anthocyanin monomers from grape pomace. Food Chem 310: 125830.

8. Shameli K, Bin Ahmad M, Zamanian A, Sangpour P, Shabanzadeh P, et al. (2012) Green biosynthesis of silver nanoparticles using Curcuma longa tuber powder. Int J Nanomedicine 7: 5603-5610.

9. KB N, HH P (2015) Homogeneous catalytic activity of gold nanoparticles synthesized using turnip (Brassica rapa L.) leaf extract in the reductive degradation of cationic azo dye. Korean J Chem Eng 32: 1273-1277.

10. Baruwati B, Varma RS (2009) High value products from waste: Grape pomace extract-a three-in-one package for the synthesis of metal nanoparticles. Chem Sus Chem 2(11): 1041-1044.

11. Singh R, Shedbalkar UU, Wadhwani SA, Chopade BA (2015) Bacteriagenic silver nanoparticles: synthesis, mechanism, and applications. Appl Microbiol Biotechnol 99(11): 4579-4593.

12. Gielen B, Jordens J, Thomassen LCJ, Braeken L, Van Gerven T (2017) Agglomeration control during ultrasonic crystallization of an active pharmaceutical ingredient. Crystals 7(2): 40. 